\title{
ASYMPTOTIC BEHAVIOR OF SOLUTIONS OF PERTURBED LINEAR SYSTEMS
}

\author{
L. E. BOBISUD
}

Abstract. The existence of solutions of the system $y^{\prime}+A y=$ $f(t, y)$ having the form $y(t)=Z(t) a(t)$ is proved, where $Z(t)$ satisfies $Z^{\prime}+A Z=0$ and the vector $a(t)$ has limit $\alpha$ as $t$ increases. Estimates for the rate of convergence to zero of $a(t)-\alpha$ and of $y(t)-Z(t) \alpha$ are obtained.

Let $Z(t)$ be a fundamental matrix of solutions for

$$
z^{\prime}+A z=0,
$$

where $z$ is an $n$-vector and $A$ is an $n \times n$ matrix of constants. We shall be concerned with the possibility of writing solutions of

$$
y^{\prime}+A y=f(t, y)
$$

$(y, f-n$-vectors $)$ in the form

$$
y(t)=Z(t) a(t)
$$

where the vector $a(t)$ has (finite) limit $\alpha$ as $t \rightarrow \infty$. Our conditions will be such that both $a(t)-\alpha$ and $y(t)-Z(t) \alpha$ converge to zero, and we obtain estimates on the rates of convergence.

Problems of this nature have been investigated ([4]-[7], [10]-[13]) for $n$th order differential equations; of these papers [4], [5], [13] assume that the linear differential equation corresponding to (1) is disconjugate, and are thus able to obtain more precise results. Only [1], [2], [4], [9] seem to have considered the more general question of systems. The present results are related to these; however, we are able to obtain results for a more general class of functions $f$ (see Corollary 2 and the example preceding it), and our asymptotic estimates are better. The latter is accomplished, in part, by estimating separately each component of $\boldsymbol{f}$ and by restricting consideration to constant matrices $A$.

For an $n$-vector $\boldsymbol{a}$ we use the norm $\|\boldsymbol{a}\|=\sum_{i=1}^{n}\left|a_{i}\right|$, we use also the notations $|\boldsymbol{a}|=\left(\left|a_{1}\right|,\left|a_{2}\right|, \cdots,\left|a_{n}\right|\right)$ and $\mathbf{1}=(1,1, \cdots, 1)$. For two $n$ vectors $\boldsymbol{p}, \boldsymbol{q}$ we write $\boldsymbol{p} \geqq \boldsymbol{q}$ if $p_{1} \geqq q_{1}, p_{2} \geqq q_{2}, \cdots, p_{n} \geqq q_{n}$. For an $n \times n$

Received by the editors March 1, 1971 and, in revised form, February 15, 1972. AMS 1970 subject classifications. Primary 34E05, 34E10.

Key words and phrases. Asymptotic behavior, perturbations. 
matrix $B=\left(b_{i j}\right)$ we shall form an $n$-vector $\|B\|$ with components $\|B\|_{i}=$ $\sup _{1 \leqq j \leqq n}\left|b_{i j}\right|$, and we shall denote by $|B|$ the matrix $\left(\left|b_{i j}\right|\right)$. Note that we have $|B a| \leqq\|B\| \cdot\|\boldsymbol{a}\|$.

THEOREM. Suppose for a given vector $\alpha$ there exist $T>0$, a fundamental matrix $Z(t)$ of (1), and functions $g, \psi$ with the following properties:

(i) $f$ is continuous on $[T, \infty) \times R^{n}$;

(ii) $\boldsymbol{g}(t, s, \boldsymbol{x})$ is continuous on $[T, \infty) \times[T, \infty) \times R^{n}, 0 \leqq g(t, s, p) \leqq$ $\boldsymbol{g}(t, s, q)$ whenever $0 \leqq p \leqq q$, and $g$ satisfies $\left|Z^{-1}(t) f(s, y)\right| \leqq g(t, s,|y-Z(s) \alpha|)$ for $(t, s, y) \in[T, \infty) \times[T, \infty) \times R^{n}$;

(iii) $\psi \in C([T, \infty)), \psi>0, \lim _{t \rightarrow \infty} \psi(t)=0$, and

on $[T, \infty)$.

$$
\psi(t) \geqq \int_{t}^{\infty} g(s, s,|Z(s)| \psi(s)) d s
$$

Then there exist solutions $y$ of (2) of the form (3) where $\lim _{t \rightarrow \infty} a(t)=\alpha$, and we have

on $[T, \infty)$.

$$
\begin{gathered}
|a(t)-\alpha| \leqq \psi(t), \\
|y(t)-Z(t) \alpha| \leqq \int_{t}^{\infty} g(s-t, s,|Z(s)| \psi(s)) d s
\end{gathered}
$$

Proof. Define an operator $F$ on $\mathscr{A} \equiv\{x(t) \in C([T, \infty)):|x(t)-\alpha| \leqq$ $\psi(t)$ for $t \geqq T\}$ by

$$
F \boldsymbol{x}(t)=\alpha-\int_{t}^{\infty} Z^{-1}(s) f(s, Z(s) \boldsymbol{x}(s)) d s ;
$$

we shall use the Schauder-Tychonov theorem [2] to prove that $F$ has a fixed point in $\mathscr{A}$. Since for $x \in \mathscr{A}$

$$
\begin{aligned}
\left|\int_{t}^{\infty} Z^{-1}(s) f(s, Z(s) x(s)) d s\right| & \leqq \int_{t}^{\infty} g(s, s,|Z(s)[x(s)-\alpha]|) d s \\
& \leqq \int_{t}^{\infty} g(s, s,|Z(s)| \Psi(s)) d s \leqq \psi(t),
\end{aligned}
$$

$F$ is indeed defined on $\mathscr{A}$ and maps $\mathscr{A}$ into $\mathscr{A}$. It is easily seen in a similar manner that $F$ is continuous, i.e., if $\boldsymbol{x}_{n} \in \mathscr{A}$ and $\boldsymbol{x}_{n} \rightarrow \boldsymbol{x}$ uniformly on compact subsets of $[T, \infty)$, then $F x_{n} \rightarrow F x$ uniformly on compact subsets of $[T, \infty)$. Since the functions in $\mathscr{A}$ are bounded, it remains only to show that the functions of $F \mathscr{A}$ are equicontinuous. Let $x \in \mathscr{A}$ and $t_{1} \geqq t_{2} \geqq T ;$ then

$$
\left|F \boldsymbol{x}\left(t_{1}\right)-F \boldsymbol{x}\left(t_{2}\right)\right| \leqq \int_{t_{2}}^{t_{1}}\left|Z^{-1}(s) f(s, Z(s) \boldsymbol{x}(s))\right| d s \leqq \int_{t_{2}}^{t_{1}} \boldsymbol{g}(s, s,|Z(s)| \Psi(s)) d s,
$$

which is small if $\left|t_{1}-t_{2}\right|$ is since $g$ is continuous. 
Let $a(t) \in \mathscr{A}$ be a fixed point of $F$; then $y(t)=Z(t) a(t)$ is a solution of (2) on $[T, \infty)$, and (4) is established. To prove (5) observe that

$$
\begin{aligned}
|\boldsymbol{y}(t)-Z(t) \boldsymbol{\alpha}| & =\left|Z(t) \int_{t}^{\infty} Z^{-1}(s) f(s, Z(s) a(s)) d s\right| \\
& \leqq \int_{t}^{\infty}\left|Z^{-1}(s-t) f(s, Z(s) a(s))\right| d s \\
& \leqq \int_{t}^{\infty} g(s-t, s,|Z(s)| \psi(s)) d s
\end{aligned}
$$

Two subcases of this theorem are of particular interest, and we discuss them as corollaries. The first deals with the case where $\left\|Z^{-1}(t) f(t, Z(t) a(t))\right\|$ is integrable for any bounded continuous $\boldsymbol{a}$.

CoRollary 1. Suppose for some fundamental matrix $Z(t)$ of (1) there exists a constant $M$ and a continuous function $h$ on $[0, \infty)^{n+2}$ such that

(i) $0 \leqq \boldsymbol{h}(t, s, \boldsymbol{p}) \leqq \boldsymbol{h}(t, s, \boldsymbol{q})$ whenever $0 \leqq \boldsymbol{p} \leqq \boldsymbol{q}$;

(ii) $\left|Z^{-1}(t) f(s, y)\right| \leqq h(t, s,|y|)$;

(iii) $\int_{0}^{\infty} \boldsymbol{h}(s, s, M\|Z(s)\|) d s<\infty$.

Then for all $\alpha \in R^{n}$ such that $\|\alpha\|<\frac{1}{2} M$ the hypotheses of the theorem are satisfied with

$$
\begin{aligned}
\boldsymbol{g}(t, s, \boldsymbol{p}) & \equiv \boldsymbol{h}\left(t, s, \boldsymbol{p}+\frac{1}{2} M\|Z(s)\|\right) \\
\psi(t) & \equiv \int_{t}^{\infty} \boldsymbol{g}\left(s, s, \frac{1}{2} M\|Z(s)\|\right) d s
\end{aligned}
$$

Proof. It is necessary to verify (ii) and (iii) of the theorem. But $\left|Z^{-1}(t) \boldsymbol{f}(s, \boldsymbol{y})\right| \leqq \boldsymbol{h}(t, s,|\boldsymbol{y}-Z(s) \boldsymbol{\alpha}|+\|Z(s)\| \cdot\|\boldsymbol{\alpha}\|) \leqq \boldsymbol{g}(t, s,|\boldsymbol{y}-Z(s) \boldsymbol{\alpha}|)$, verifying (ii). Choosing $T$ so large that $\int_{T}^{\infty} \boldsymbol{h}(s, s, M\|Z(s)\|) d s<(M / 2 n) \mathbf{1}$, and observing that $g$ is increasing in its last argument, we have

$$
\Psi(t)=\int_{t}^{\infty} \boldsymbol{g}\left(s, s, \frac{1}{2} M\|Z(s)\|\right) d s=\int_{t}^{\infty} \boldsymbol{h}(s, s, M\|Z(s)\|) d s<\frac{M}{2 n} \mathbf{1},
$$

so $\|\psi(t)\|<\frac{1}{2} M$, and

$$
\int_{t}^{\infty} \boldsymbol{g}(s, s,|Z(s)| \Psi(s)) d s \leqq \int_{t}^{\infty} \boldsymbol{g}\left(s, s, \frac{1}{2} M\|Z(s)\|\right) d s=\psi(t) .
$$

We remark that the error estimate (5) can be replaced by the weaker but more convenient statement

$$
|\boldsymbol{y}(t)-Z(t) \boldsymbol{\alpha}| \leqq \int_{t}^{\infty} \boldsymbol{g}\left(s-t, s, \frac{1}{2} M\|Z(s)\| \mid\right) d s .
$$


This corollary is connected with some results of [2], [4], [5], [7], [11], [12] when specialized to the $n$th order linear differential equation

$$
x^{(n)}=f\left(t, x, x^{\prime}, \cdots, x^{(n-1)}\right)
$$

where $f$ satisfies $\left|f\left(t, x, \cdots, x^{(n-1)}\right)\right| \leqq \sum_{i=0}^{n-1} g_{i}(t)\left|x^{(i)}\right|^{r_{i}}$ with $r_{i}>0$ and (8) $\int_{0}^{\infty} t^{n-1}\left(1+t+\cdots+t^{n-i-1}\right)^{r_{i}} g_{i}(t) d t<\infty \quad(i=0, \cdots, n-1)$.

Indeed, writing (7) as a first-order system in the usual way (cf. [2]), we obtain the result that for any $\alpha$ there exists a solution $x_{\alpha}$ of (7) such that

$$
\begin{aligned}
& \left|x_{\alpha}^{(j)}-\alpha_{j+1}-t \alpha_{j+2}-\cdots-\left(t^{n-j-1} /(n-j-1) !\right) \alpha_{n}\right| \\
& \leqq \int_{t}^{\infty}(s-t)^{j}\left\{\sum_{i=0}^{n-1}\|\alpha\|^{r_{i}}\left(1+s+\cdots+s^{n-i-1}\right)^{r_{i}} g_{i}(s)\right\} d s \\
& \quad(j=0, \cdots, n-1) .
\end{aligned}
$$

We turn now to a different application of our main result. Consider the example of (2) given by the single first-order equation

$$
y^{\prime}=f(t, y) \equiv(1 / t)(y-1)^{2}+1 / t^{3} ;
$$

with $\alpha=1, Z(t)=1$, we have $g(t, s,|y-\alpha|)=(1 / t)|y-1|^{2}+1 / t^{3}$. Since $(1 / t)|y(t)-1|^{2}$ is not necessarily integrable even when $\lim _{t \rightarrow \infty} y(t)=1$, Corollary 1 is not applicable, and neither are the results of [2], [4]. However, out main theorem can be applied with $\psi(t)=t^{-2+\varepsilon}$ for any $\varepsilon>0$, as is readily seen, and from (5) we conclude that there exist solutions $y(t)$ of (9) such that $|y(t)-1| \leqq 1 / t^{2}$. In this example the right-hand side $f$ has the form

$$
f(t, y)=f_{1}(t, y-Z(t) \alpha)+f_{2}(t, y),
$$

where $f_{2}\left(=t^{-3}\right)$ is integrable for bounded $y$ but $f_{1}$ is not integrable even for all $\boldsymbol{y}$ tending to $Z(t) \boldsymbol{\alpha}$. We generalize this situation in the following corollary.

COROllary 2. Suppose for some fundamental matrix $Z(t)$ of (1) and some $\alpha$ we have (10) valid, where there exist continuous functions $\boldsymbol{h}_{1}, \boldsymbol{h}_{2}$ on $[0, \infty)^{n+2}$ satisfying

(i) $0 \leqq \boldsymbol{h}_{i}(t, s, \boldsymbol{p}) \leqq \boldsymbol{h}_{i}(t, s, \boldsymbol{q})$ if $0 \leqq \boldsymbol{p} \leqq \boldsymbol{q}(i=1,2)$;

(ii) $\left|Z^{-1}(t) f_{2}(s, y)\right| \leqq h_{2}(t, s,|y|)$, with

$$
\int_{0}^{\infty} \boldsymbol{h}_{2}(s, s,\|\mathrm{Z}(s)\| \cdot\|\boldsymbol{\alpha}\|) d s<\infty ;
$$

(iii) $\left|Z^{-1}(t) f_{1}(s, y)\right| \leqq h_{1}(t, s,|y-Z(s) \boldsymbol{\alpha}|)$. 
Suppose finally that there exists a continuous function $\boldsymbol{\phi}(t)>0$ satisfying, for some $\varepsilon>0$ and all large $t, \phi(t) \rightarrow 0$ as $t \rightarrow \infty$,

$$
\begin{gathered}
\boldsymbol{\phi}(t) \geqq \int_{t}^{\infty} \boldsymbol{h}_{1}(s, s,(1+\varepsilon)|Z(s)| \boldsymbol{\phi}(s)) d s, \\
\int_{t}^{\infty} \boldsymbol{h}_{2}(s, s, 2\|Z(s)\| \cdot\|\boldsymbol{\alpha}\|) d s \leqq \varepsilon \boldsymbol{\phi}(t) .
\end{gathered}
$$

Then the hypotheses of the theorem are satisfied for large $T$ with

$$
\begin{gathered}
g(t, s, p)=h_{1}(t, s, p)+h_{2}(t, s, p+\|Z(s)\| \cdot\|\alpha\|), \\
\psi(t)=\phi(t)+\int_{t}^{\infty} h_{2}(s, s, 2\|Z(s)\| \cdot\|\alpha\|) d s .
\end{gathered}
$$

Proof. Hypothesis (ii) of the theorem is clearly satisfied; we have only to verify (iii). But for sufficiently large $t$,

$$
\begin{aligned}
\int_{t}^{\infty} \boldsymbol{g}(s, s,|Z(s)| \psi(s)) d s \leqq & \int_{t}^{\infty} \boldsymbol{h}_{1}(s, s,(1+\varepsilon)|Z(s)| \boldsymbol{\phi}(s)) d s \\
& +\int_{t}^{\infty} \boldsymbol{h}_{2}(s, s,|Z(s)| \psi(s)+\|Z(s)\| \cdot\|\boldsymbol{\alpha}\|) d s \\
\leqq & \phi(t)+\int_{t}^{\infty} \boldsymbol{h}_{2}(s, s, 2\|Z(s)\| \cdot\|\boldsymbol{\alpha}\|) d s=\psi(t) .
\end{aligned}
$$

As an application of Corollary 2 we determine conditions on the coefficients of the differential equation

$$
u^{(n)}=\sum_{i=0}^{n-1} a_{i}(t)\left(u^{(i)}\right)^{r_{i}}+b(t)
$$

$\left(r_{i}>1\right)$ which guarantee the existence of a solution $u(t)$ such that $u^{(j)}(t) \rightarrow 0$ as $t \rightarrow \infty \quad(j=0,1, \cdots, n-1)$. If $b$ and all the $a_{i}$ are integrable, this follows from Corollary 1 , hence we assume that not all $a_{i}$ are integrable. Writing the equation as a system in the usual manner, we have

$$
Z(t)=\left[\begin{array}{ccccc}
1 & t & t^{2} / 2 ! & \cdots & t^{n-1} /(n-1) ! \\
0 & 1 & t & \cdots & t^{n-2} /(n-2) ! \\
\cdot & \cdot & \cdot & & \cdot \\
\cdot & \cdot & \cdot & & \cdot \\
\cdot & \cdot & \cdot & & \cdot \\
0 & 0 & 0 & \cdots & 1
\end{array}\right]
$$


for fundamental matrix and $\alpha=0$. With

$$
\begin{aligned}
& h_{2, k}(t, s,|y|)=\frac{t^{n-k}}{(n-k) !}|b(s)| \\
& h_{1, k}(t, s,|y|)=\frac{t^{n-k}}{(n-k) !} \sum_{i=0}^{n-1}\left|a_{i}(s)\right|\left|y_{i+1}\right|^{r_{i}}
\end{aligned}
$$

we have that $\phi$ must satisfy

$$
\phi_{k}(t) \geqq \int_{t}^{\infty} \frac{s^{n-k}}{(n-k) !} \sum_{i=0}^{n-1}\left|a_{i}(s)\right|(1+\varepsilon)^{r_{i}}\left[\sum_{j=i}^{n} \frac{s^{j-i}}{(j-i) !} \phi_{j}(s)\right]^{r_{i}} d s .
$$

For convenience let $C_{i}=\sum_{j=1}^{n}((n-1) ! /(j-1) !(n-j) !)$ and $r=\max r_{i}$. Let $\phi_{1}(t)$ be any solution of

$$
\phi_{1}(t) \geqq(1+\varepsilon)^{r} \int_{t}^{\infty} \frac{s^{n-1}}{(n-1) !} \sum_{i=0}^{n-1}\left|a_{i}(s)\right|\left[\frac{C_{i}}{s^{i-1}} \phi_{1}(s)\right]^{r_{i}} d s
$$

and define

$$
\phi_{j}(t)=\frac{(n-1) !}{(n-j) ! t^{j-1}} \phi_{1}(t) \quad(j=2, \cdots, n) .
$$

Then

$$
\begin{aligned}
\phi_{j}(t) & \geqq(1+\varepsilon)^{r} \int_{t}^{\infty} \frac{s^{n-j}}{(n-j) !} \sum_{i=0}^{n-1}\left|a_{i}(s)\right|\left[\frac{C_{i}}{s^{i-1}} \phi_{1}(s)\right]^{r_{i}} d s \\
& \geqq \int_{t}^{\infty} \frac{s^{n-j}}{(n-j) !} \sum_{i=0}^{n-1}\left|a_{i}(s)\right|(1+\varepsilon)^{r_{i}}\left[\sum_{k=i}^{n} \frac{s^{k-i}}{(k-i) !} \phi_{k}(s)\right]^{r_{i}} d s,
\end{aligned}
$$

as required. It thus remains to show that (12) has a suitable solution. Suppose $0 \leqq \phi_{1}(t) \leqq 1$, and let $q=\min r_{i}>1$, so $\phi_{1}^{r_{i}} \leqq \phi_{1}^{q}$; then if $\phi_{1}$ satisfies

$$
\phi_{1}(t) \geqq \int_{t}^{\infty} \frac{(1+\varepsilon)^{r}}{(n-k) !} s^{n-1} \sum_{i=0}^{n-1}\left|a_{i}(s)\right|\left(\frac{C_{i}}{s^{i-1}}\right)^{r_{i}} \phi_{1}^{q}(s) d s \equiv \int_{t}^{\infty} k(s) \phi_{1}^{q}(s) d s,
$$

it will also satisfy (12). The equality in (13) can be solved to yield

$$
\phi_{1}(t)=\left\{\left[\phi_{1}\left(t_{0}\right)\right]^{1-q}+(q-1) \int_{t_{0}}^{t} k(s) d s\right\}^{-1 /(q-1)}
$$

provided $\int^{\infty} k(s) d s=\infty$ and the positive constant $\phi_{1}\left(t_{0}\right)$ is chosen less than 1. Thus (11) has solutions tending to zero with their first $n-1$ derivatives if

$$
\int \sum_{i=0}^{t n-1}\left|a_{i}(s)\right| s^{r_{i}(1-i)+n-1} d s \rightarrow \infty
$$

as $t \rightarrow \infty$ and $b(t)$ satisfies $\int_{t}^{\infty} s^{n-1}|b(s)| d s \leqq \varepsilon(n-1) ! \phi_{1}(t)$ for some $\varepsilon>0$. 
I wish to thank the referee for pointing out an error in an earlier version and for improvement in the statement of the theorem.

\section{REFERENCES}

1. J. W. Bebernes and N. X. Vinh, On the asymptotic behavior of linear differential equations, Amer. Math. Monthly 72 (1965), 285-287. MR 31 \#6011.

2. F. Brauer and J. S. W. Wong, On asymptotic behavior of perturbed linear systems, J. Differential Equations 6 (1969), 142-153. MR 39 \#570.

3. W. A. Coppel, Stability and asymptotic behavior of differential equations, Heath, Boston, Mass., 1965. MR 32 \#7875.

4. J. K. Hale and N. Onuchic, On the asymptotic behavior of solutions of a class of differential equations, Contributions to Differential Equations 2 (1963), 61-75. MR 28 \#2281.

5. T. G. Hallam, Asymptotic behavior of the solution of an nth order nonhomogeneous ordinary differential equation, Trans. Amer. Math. Soc. 122 (1966), 177-194. MR 32 \#6000.

6. I. N. Katz, Asymptotic behavior of solutions to some nth order linear differential equations, Proc. Amer. Math. Soc. 21 (1969), 657-662.

7. P. Locke, On the asymptotic behavior of solutions of an nth-order nonlinear equation. Proc. Amer. Math. Soc. 18 (1967), 383-390. MR 35 \#3167.

8. - Correction to a paper by Brauer and Wong, J. Differential Equations 9 (1971), 380. MR 42 \#7987.

9. M. Ráb, Les formules asymptotiques pour les solutions d'un système des équations différentielles, Arch. Math. (Brno) 1 (1965), 199-212. MR 33 \#6026.

10. W. F. Trench, On the asymptotic behavior of solutions of second order linear differential equations, Proc. Amer. Math. Soc. 14 (1963), 12-14. MR 26 \#412.

11. P. Waltman, On the asymptotic behavior of solutions of a nonlinear equation, Proc. Amer. Math. Soc. 15 (1964), 918-923. MR 31 \#445.

12. - On the asymptotic behavior of solutions of an nth order equation, Monatsh. Math. 69 (1965), 427-430. MR 32 \#2686.

13. D. Willett, Asymptotic behavior of disconjugate nth order differential equations, Canad. J. Math. 23 (1971), 293-314.

Department of Mathematics, University of Idaho, Moscow, Idaho 83843 